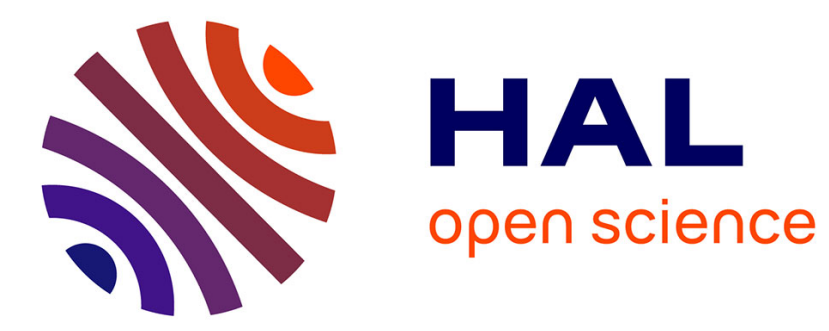

\title{
Physical and Perceptual Independence of Ultrasonic Vibration and Electrovibration for Friction Modulation
}

Eric Vezzoli, Wael Ben Messaoud, Michel Amberg, Betty Lemaire-Semail, Frédéric Giraud, Marie-Ange Bueno

\section{> To cite this version:}

Eric Vezzoli, Wael Ben Messaoud, Michel Amberg, Betty Lemaire-Semail, Frédéric Giraud, et al.. Physical and Perceptual Independence of Ultrasonic Vibration and Electrovibration for Friction Modulation. IEEE Transactions on Haptics (ToH), 2015, 10.1109/TOH.2015.2430353 . hal-01253575

\section{HAL Id: hal-01253575 \\ https://inria.hal.science/hal-01253575}

Submitted on 11 Jan 2016

HAL is a multi-disciplinary open access archive for the deposit and dissemination of scientific research documents, whether they are published or not. The documents may come from teaching and research institutions in France or abroad, or from public or private research centers.
L'archive ouverte pluridisciplinaire HAL, est destinée au dépôt et à la diffusion de documents scientifiques de niveau recherche, publiés ou non, émanant des établissements d'enseignement et de recherche français ou étrangers, des laboratoires publics ou privés. 


\title{
Physical and perceptual independence of ultrasonic vibration and electrovibration for friction modulation
}

\author{
Eric Vezzoli, Wael Ben Messaoud, Michel Amberg, Betty Lemaire-Semail, Member, IEEE, Frédéric \\ Giraud, Member, IEEE , and Marie-Ange Bueno
}

\begin{abstract}
Two different principles are available to modulate the user perceived roughness of a surface: electrovibration and ultrasonic vibration of a plate. The former enhances the perceived friction coefficient and the latter reduces it. This paper will highlight the independence of the two effects on the physical and perceptual point of view to confirm the increased range of sensation that can be supplied by the two coupled techniques. Firstly, a tribometric analysis of the induced lateral force on the finger by the two coupled effects will be presented, then a study on the dynamic of the two effects will be reported. In the end, a psychophysical experiment on the perception of the two coupled techniques will be shown.
\end{abstract}

Index Terms - Tactile devices and display, Tactile stimulator, Electrovibration, Squeeze film effect, Ultrasonic devices, Electrostatic force, Friction modulation, Dynamic system response.

\section{INTRODUCTION}

$\mathrm{T}$ the last years, a big interest has raised around the touch sense in the human-machine interaction, due to the relevance of this sense in the human perception through the identification and manipulation of objects. In the consumer world, the lack of the feedback of a real keyboard was one of the main issues during the spreading of the tactile screen technology in the beginning of the smartphone era. Nowadays, the tactile feedback problem is faced with the vibration of the device, which provides the user with some information, but is ineffective to simulate the tactile perception of the visualized surface on the screen. Nevertheless, a better immersion into virtual reality and a more efficient remote control will arise with the development of optimized tactile stimulation techniques.

There are different approaches for the tactile simulation technique mediated by a flat surface: it is possible to simulate textures with controlled vibrations of a plate [1] which recreate on the finger

- E.V. Author is with L2EP-IRCICA Laboratory, University of Lille 1, Lille, France, 59650. E-mail:eric.vezzoli@ed.univ-lille1.fr.

- W.B.M. Author is with L2EP-IRCICA Laboratory, University of Lille 1, Lille, France, 59650 and LPMT Laboratory, Ecole Nationale Supérieure d'Ingénieurs Sud Alsace, University of Haute Alsace, 68093 Mulhouse, France: E-mail: wael.ben-messaoud@ed.univ-lille1.fr

- M.A. Author is with L2EP-IRCICA Laboratory, Lille, University of Lille 1, France, 59650. E-mail: Michel.Amberg@ univ-lille1.fr.

- B.L.S. Author is with L2EP-IRCICA Laboratory, University of Lille 1, Lille, France, 59650.E-mail: betty.semail@polytech-lille.fr.

- F.G Author is with L2EP-IRCICA Laboratory, University of Lille 1, Lille, France, 59650. E-mail: Frederic.giraud@polytech-lille.fr

- M.A.B. is with LPMT Laboratory, Ecole Nationale Supérieure d'Ingénieurs Sud Alsace, University of Haute Alsace, 11 rue Alfred Werner, 68093 Mulhouse, France.E-mail: marie-ange.bueno@uha.fr the pattern of vibration induced by the real texture. Then it is possible to control the friction coefficient between an active surface and a finger. Two techniques can be coupled with position sensing technology: the ultrasonic vibration of a plate and the electrovibration. The first is able to reduce the friction coefficient perceived by the user due to the creation of a film of pressurized air between the finger and the plate [2][3][4], while the second increases it by the induced electrostatic force between the finger and the polarizing plate [4][5][7][8]. Both of these technologies involve a global stimulation of the whole fingertip area touching the surface. The coupling of these technologies with finger position sensing technique allows establishing a spatio-temporal relation leading to the possibility of fine texture simulation [2][3][9].

The earliest attempt to couple ultrasonic vibrations and electrovibration was performed by Giraud et al. [10], confirming the technical possibility to use them together. The range of sensation can be enlarged due to their opposite influence on the perceived friction allowing a better rendering of simulated texture. The aim of this study is to show that the two principles can be added linearly in the static, dynamic and perceptual points of view. In this paper, both technologies will be explained with references to current models and the development of the haptic interfaces will be analyzed; then, the independence of the two tactile stimulation techniques will be shown in the static regime and an analysis of their dynamic behavior will be performed. In the end, a psychophysical experiment showing the linearity of the two effects in the perceptual point of view will be presented. 


\section{Haptic StimUlator}

In this section, the two different tactile stimulators used in the experiments will be presented. The standalone stimulator will be used for static measure and psychophysical evaluation, because it performs simultaneously the two effects; whereas the fast response simulator will be employed in the dynamic behavior study.

\subsection{Standalone stimulator}

The haptic stimulator used for the static and the psychophysical experience is based on a modified USBstandalone version of the STIMTAC [9]. STIMTAC works according to ultrasonic vibrations of a plate, which can reduce friction. The tactile plate used in this experiment has a resonant frequency of $38220 \mathrm{~Hz}$ at ambient temperature. In the modified version, the stimulator has the inverter electrically uncoupled from the vibrating plate. In this way, it was feasible to introduce an amplifier to apply a high voltage to the tactile plate inducing the electrovibration effect. The elecrovibration force can be expressed as:

$$
F_{e}=\varepsilon_{0} A v_{e}^{2} /\left[2\left(\frac{T_{i}}{\varepsilon_{i}}+\frac{T_{s}}{\varepsilon_{s}}\right)\left(T_{s}+T_{i}\right)\right]
$$

where $\varepsilon_{0}$ is the vacuum permittivity, $A$ is the finger area in contact with the polarizing plate, $T_{i}$ and $T_{s}$ are the thicknesses of the insulator and the stratum corneum, $\varepsilon_{i}$ and $\varepsilon_{s}$ are the relative permittivities of the insulator and stratum corneum and $v_{e}$ is the effective voltage acting on the finger filtered by the human skin [5]. The utilized amplifier (UVA2kV, Ultravolt, USA) coupled with the signal generator inside is designed to provide a programmable voltage modulated at $440 \mathrm{~Hz}$. In particular, it is possible to express the lateral force, $F_{t}$, felt by the finger in an hybrid stimulator as:

$F_{t}=(\mu-\Delta \mu)\left(F_{n}+F_{e}\right)$

where $\mu$ is the friction coefficient between the finger and the plate, $\Delta \mu$ is the induced variation of the friction coefficient performed by ultrasonic vibrations [2], $F_{n}$ is the normal force applied by the finger and $F_{e}$ is the induced normal force between the finger and the plate by electrostatic force from (1). To derive this formula, it is assumed that there is no mutual influence between the friction modulation induced by electrovibration and

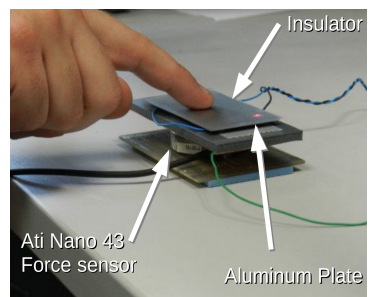

a

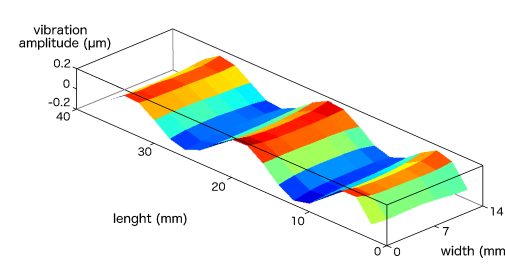

b
Fig. 1. a. Static measurement system. The red spot on the plate is the focus of the laser of the vibrometer. b. cartography of the fast response stimulator. ultrasonic vibrations.

For static measure, in order to monitor the forces and the friction coefficient, a 6 axis force sensor (nano 43, ATI, USA) was used to ensure a resolution of $7.8 \mathrm{mN}$ with a sampling period of $400 \mu \mathrm{s}$. To measure the vibration amplitude, an interferometer vibrometer was focused on the plate. To perform static measure, the tactile plate has been unmounted from the tactile stimulator and placed on the force sensor, figure 1a. At last, data are acquired with an oscilloscope (3014B, Tektronik, USA) then handled.

\subsection{Fast response stimulator}

To perform the dynamic behavior measurement, a specific, tactile stimulator has been designed to ensure a fast mechanical time response of the vibrating plate. It is needed to monitor the vibration amplitude of the plate itself because it is the effective parameter responsible for the generation of friction reduction [2] [11]. This plate has been equipped with five piezoelectric ceramics, four of them are used like actuators and one of them is used as a sensor. To calibrate the relationship between the signal provided by the ceramics and the effective vibration of the plate, a laser vibrometer has been used (OVF-5000, Polytech, Germany). To guarantee consistency with the previous setup, the spatial length of the vibration mode and the plastic film used to insulate for electrovibration are the same as the plate used for static measure, figure $1 \mathrm{~b}$. It may be noted that this plate was not designed to stimulate the finger with the two different technologies simultaneously.

\section{Physical Coupling}

\subsection{Static Independence}

In this section, it will be demonstrated that there is no influence between the two tactile stimulation techniques while coupled. Electrovibration and friction reduction in ultrasonic devices act in opposition: electrovibration increases the normal force between the pulp of the finger and the plate whereas in the ultrasonic devices a reduction of the friction is experienced, see equation (2). The friction force and the normal force of a finger sliding on a tactile simulator have been recorded while the effects have been coupled with different phase shifts. The participant gave his informed consent to perform the experiment. This one has been carried out in free exploration by the user but data have been recorded after a period of training to assume the normal force constant as well as the speed. Before the experience, the subject was asked to wash his hands and completely dry them with talcum powder. To avoid the deposition of moisture on the plate, the exploration was allowed just 30 seconds before having a pause of 30 seconds to let the finger dry. Visual feedback of the normal force applied on the plate was provided by the oscilloscope. The experiment was performed with 2 different phase shifts between the two effects (0 and 90 degrees) to study different possible interactions between the 2 principles, figure 2 . It may be noted that before this measurement, the effect was 

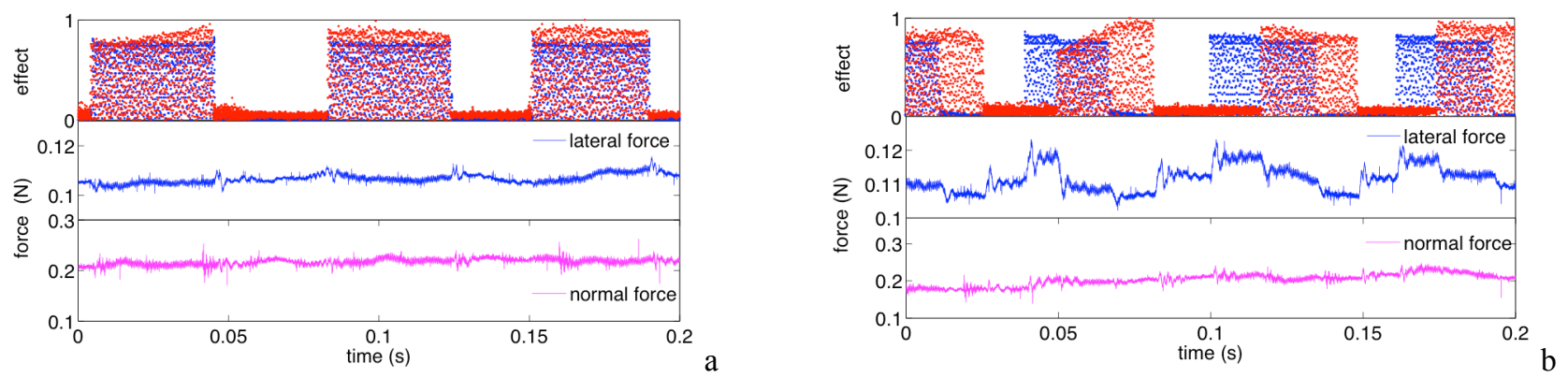

Fig. 2. Example of measure of coupling of electrovibration and ultrasonic vibrations in phase $\mathbf{a}$, and in quadrature $\mathbf{b}$. In the first graph is reported the presence of the effect, electrovibration (blue) and ultrasonic vibrations (red). In the second graph is reported the recorded lateral force. In the third graph is reported the measured normal force.

singularly tuned on the participant finger to induce a similar reduction/increasing of the lateral force to highlight the possible mutual influence.

Figure $2 \mathrm{a}$ represents the case where the electrovibration effect and the ultrasonic vibrations are applied in phase. For a constant normal force applied by the finger while sliding, it has been recorded a constant lateral friction force.

On a different phase (90 degrees) between the two friction modulation techniques, a stair modulation appears on the measured lateral force, figure $2 b$.

From all the performed measurements (about 50 tries), the friction coefficient has been computed for each considered case: electrovibration applied, ultrasonic vibration applied, no friction modulation and both effects applied. The average on the performed measurements is reported in figure 3 . These measurements substantially confirm that the two techniques are fully mixable and that there is no mutual influence between the two in the static regime on the tribological point of view. Moreover, that is possible to find a level of the two effects to fully compensate each other and the resulting induced modulation of the lateral force.

\subsection{Dynamic behavior}

In this section, the possibility to couple the two effects also on a dynamic point of view will be discussed. For this experiment, the fast response simulator presented in section 2.2 was used. To compare the two effects, their rise time has been measured. This one is defined by the delay measured between the $10 \%$ and the $90 \%$ of the equilibrium value of the tangential force measure, after the application of the excitation. To perform this experiment, the tribometer detailed in [5] has been used due the larger bandwidth $(10 \mathrm{kHz})$. A similar experience was performed by Meyer et al. [12]; they measured the response of the tangential force to a reference step either

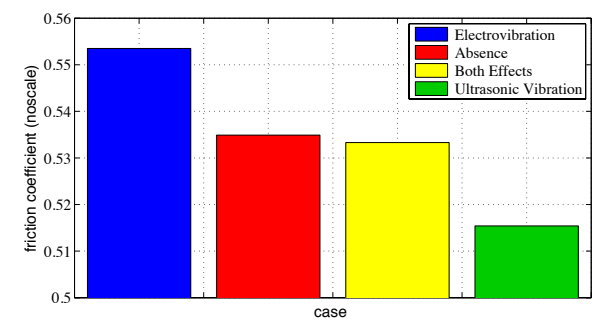

Fig. 3 Calculated $\mu$ for the different modulations in the considered cases. When the effects are regulated to induce the same friction modulation, their coupling results in a compensation of the induced friction modulation. due to ultrasonic vibrations or electrovibration, for different finger speeds. They found a higher value of the time rise of the first compared to the latter worthy to be deeply investigated. To perform this measure, it was necessary to record the parameter generating tactile stimulation: the vibration amplitude for the ultrasonic devices and the voltage applied for the electrovibration. Moreover a unique surface to exploit both experiments is required to compare the two effects. For a similar plate design than the one used in [12] we have found a mechanical time response of the vibration mode of the plate of about $15 \mathrm{~ms}$. This could be the cause of this discrepancy between the two stimulation principles. For this reason, the fast response stimulator described before has been used to conduct these experiments. This plate has a measured mechanical time response around $1.5 \mathrm{~ms}$. In figure 5 the measurements recorded on the same subject of the aforementioned analysis are presented. Each line represents the average of about 50 measurements. The tangential force response of both effects was analyzed for an increase and decrease of friction modulation. In the ultrasonic vibration related measure, the green line represents the vibration envelope of the plate, which has been measured through sensor ceramic. The voltage provided to the plate has been tuned to induce a vibration amplitude of $2 \mu \mathrm{m}$ peak to peak with the presence of the finger. To ensure a uniform mechanical behavior of the finger, the normal forces for the reported measurements are equal to $0.3 \mathrm{~N}$ with a standard deviation of $18 \%$. At this point, it was possible to proceed with the identification of the model to link the parameter generating the friction force modulation. To do so, the MATLAB toolbox for Black-Box modeling has

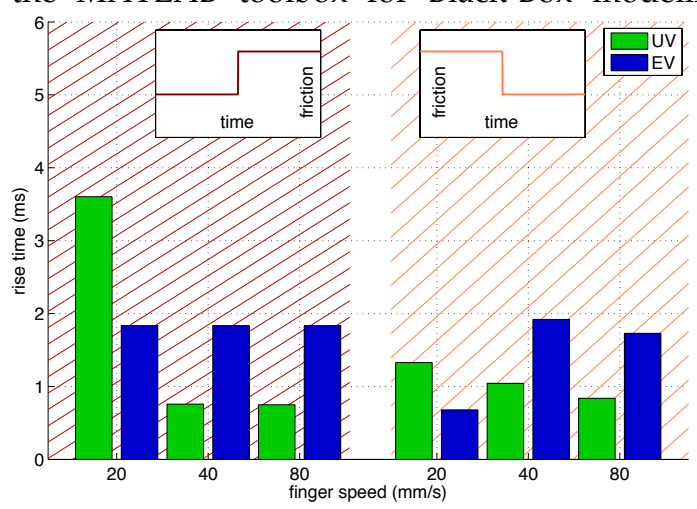

Fig. 4. Rise time of the step response for the identified models (ultrasonic vibration UV et electrovibration EV) based on the previous measures for the two different friction cases considered: mounting friction (left) and descending friction (right). 
been utilized considering a second order model. The input parameter has been the vibration amplitude envelope for the ultrasonic device model identification and the applied voltage envelope for electrovibration. For each situation, the calculated model has been evaluated with a step function to investigate the actual behavior of the effect itself. In accordance with the approach reported in [12], the parameter evaluated to compare the effect has been the rise time of the response of the identified model to the step. The results are reported in figure 4 .

\section{Perceptual Independence}

In this section, a psychophysical experiment will be presented, showing the possibility to find a level of compensation between the two effects, with which the user cannot distinguish between a surface with compensated friction modulation and a surface without any friction modulation. To perform so, the standalone stimulator was programmed to induce a friction reduction on the plate following a sinusoidal profile, with a spatial wavelength of $1 \mathrm{~cm}$. Then, it has been implemented the possibility to have the same spatial profile function with the electrovibration modulation. It was possible to adjust the electrovibration maximal voltage applied to the plate on eight different levels, from 0 to $2000 \mathrm{~V}$. The levels were equally spaced with a singular amplitude level of $287 \mathrm{~V}$. The aim of the experiment is to know if for all the users, it was possible to find a level of electrovibration where the friction modulation induced by the ultrasonic vibration was totally compensated and then the plate surface was perceived as uniform. It was asked to six subjects to freely explore the tactile plate while maintaining the normal force applied around $0.4 \mathrm{~N}$. It was necessary to introduce this limitation to ensure that the changing force did not influence the friction reduction induced by the ultrasonic
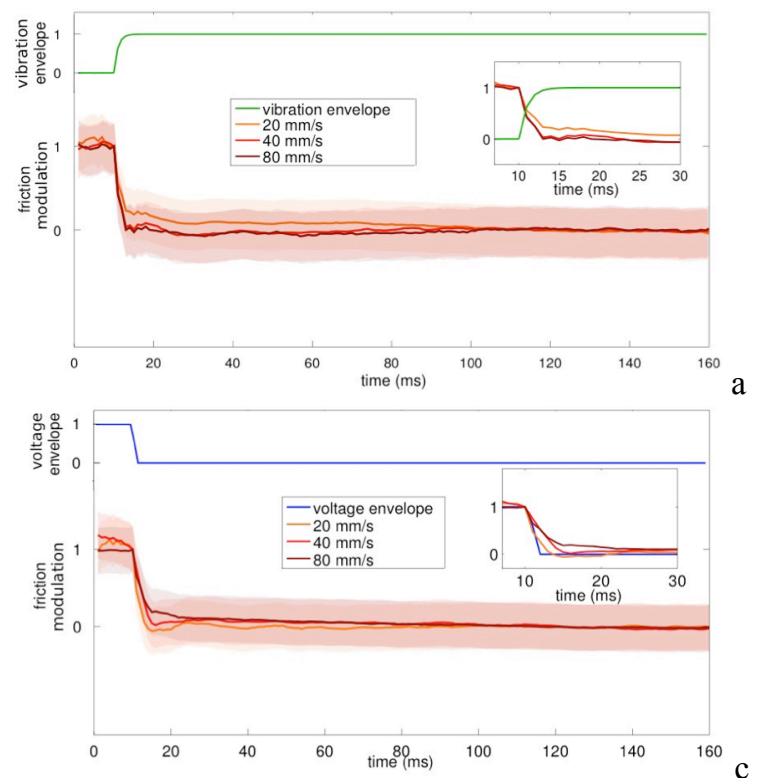

device [2] and guarantee that the vibration damping of the plate induced by the presence of the finger was stable. To avoid any noticeable difference into the dynamic of the two effects and the time response of the system that could induce distortion into the perception of the surface, it was asked to the participants to maintain a constant exploration speed (smaller than $40 \mathrm{~mm} / \mathrm{s}$ ). All of the participants gave their informed consent for the execution of the experiment. It was asked to the subjects to wear an anti-noise headset to prevent any influence of the sounds produced by the plate on their answer. The value of the normal force was provided via a graphical interface to the subjects. Before each experiment, the tactile plate was cleaned and the participants were invited to wash their hands and dry them with talcum. Before starting the experiment, the participants had the time to get accustomed to the friction modulation induced by the ultrasonic vibration with the compensation effect induced by electrovibration set to 0 (no effect of increased normal force). When the participant communicated that he was ready and exhibited a stable normal force while exploring the plate, the experiment started. The level of electrovibration was increased by one step and the subject was asked how the surface was and how it was in comparison with the set-up situation. The participant could describe the surface as non uniform or uniform, then the level of electrovibration compensation was increased by another step. To avoid moisture deposition, the exploration of the plate was allowed for 20 seconds with 20 seconds of pause to let the finger dry. After each participant, the tactile plate was cleaned to remove any remaining deposit. The starting level of compensation was always available to the users to compare the friction modulation. Every participant could find a level of electrovibration which fully compensates the friction modulation induced by the ultrasonic vibration. Whereas in other cases, the surface was perceived as non-uniform.
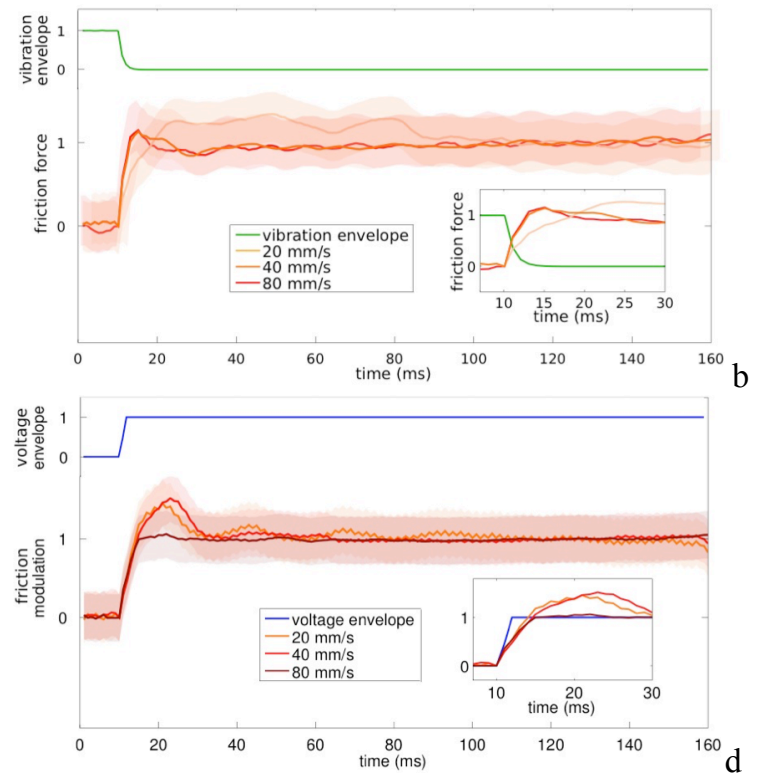

Fig. 5. Friction modulation for different finger speeds related to the measured plate vibration or voltage envelope reported in the figure. The shadowed area represents the measure standard deviation. $\mathbf{a}$, descending friction ultrasonic devices, $\mathbf{b}$ increasing friction ultrasonic devices, c decreasing friction electrovibration and $\mathbf{d}$ increasing friction electrovibration. 
After finding the level where the modulation was not perceived anymore, a different level of compensation was selected and it was asked to the participants if it was uniform or not. In case of non uniformity, the compensation level was reduced or increased by one step into the direction of the previous indicated compensation level. All the participants indicated the same level as before as the one exhibiting the uniform surface (see figure 6). The compensation level necessary to cancel the influence on the lateral force induced by the ultrasonic vibration exhibits a huge variability between the subjects. The variability of the compensation force can be explained by the variability of the thickness of the stratum corneum on the human fingertip [13] or its water content, which strongly influences the induced electrostatic force on the finger [5], as well as the possible variability of the friction modulation induced by ultrasonic vibration which has not been analyzed yet.

\section{Discussion}

The static dependence measure confirmed that it is possible to constructively couple the two effects to enhance the range of lateral force modulation experienced by the finger. Moreover, it has been demonstrated that if the influence on the lateral force by the two effects is set as similar, it is possible to obtain a friction modulation equal to zero while coupling them in phase. These measurements confirm the physical independence of the two effects and confirm the validity of the equation (2). In this range of induced modulation, it is possible to conclude that the two effects are purely additive, and there is not any mutual influence.

The measured dynamic of the two effects is an indicator of their behavior in the varying regime considering a real finger. The experiment substantially confirms that in most of the cases considered, the rising times of the two effects are comparable. Thus, they can be successfully coupled additively also in dynamic. An exception is the case of rising friction for ultrasonic vibrations with low exploration speed $(20 \mathrm{~mm} / \mathrm{s})$, this is probably due to some stick-slip effect that happened after the increasing of the friction. This phenomenon can be seen in figure $5 b$ for the lower speed of exploration. For this reason, the rising time is higher than in the other measured cases. For a hybrid tactile stimulator, it would be possible to introduce a pulse of electrovibration to increase the rising time in this low speed situation to realign the time response to the other cases and obtain a linear stimulator in dynamics. To obtain a linear coupled tactile stimulator it is necessary to design

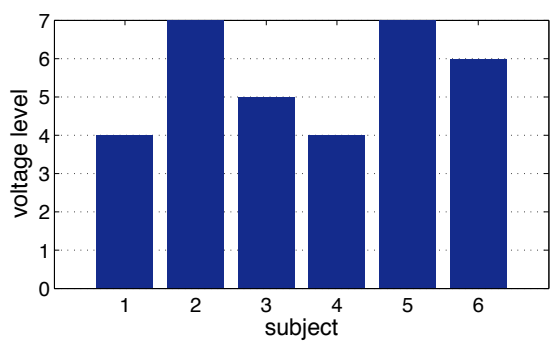

Fig.6. Compensation level of electrovibration necessary to cancel the friction modulation induced by the ultrasonic devices and have a uniform perception of the surface. the mechanical plate and chose the amplifier for the electrovibration to match the dynamic of the two effects. To obtain the effective rise time of the friction for the developing of a linearized hybrid tactile stimulator, it has to be considered the mechanical rise time of the plate for ultrasonic vibrations and the rise time of the amplifier for the electrovibration. The former is dependent on the $Q$ factor of the plate and can be reduced to 1-2 ms where the latter is just dependent on the amplifier and, with the right amplifier choice, could be neglected.

These results are obtained considering all the device parameters which affect the stimulation principle itself and are consistent between the two different techniques. As suggested by [12], fingertip mechanical behavior plays an important role in the determination of these forces and the analysis of these rising times is consistent with the analysis proposed by Wiertlewski et al. [14] for the mechanical response of the fingertip. Their analysis predicts a fingertip response in the order of milliseconds, which is in good agreement for all the considered friction modulation changes.

The psychophysical experiment on the two coupled effects confirms that it is always possible to find a level where the two effects fully compensate each other. The experiment also shows that the two friction modulations are perceived in the same way by the users. The compensation level reported in figure 6 shows that there is no difference, from the point of view of the user perception, between a real surface and a surface where the friction modulation is compensated. This does mean that the users cannot perceive the presence of the effects on the surface, but perceive just the impact of those effects on the lateral force. With these techniques, it is possible to change the friction coefficient of a surface, without influencing all the other parameter concurring to generate the user perception of the surface itself.

\section{Conclusion}

This paper analyzed the possibility to successfully couple two friction modulation techniques, electrovibration and ultrasonic vibrations, to expand the range of sensation provided to the users. An analysis of the time rise of these two effects decoupled has been proposed to analyze the possibility to couple them also in the dynamic domain. The psychophysical evaluation confirms that the effects are fully mixable and the user cannot distinguish the presence of the two compensated effects on the surface to the nonmodulated one because he/she perceives this surface as uniform.

\section{ACKNOWLEDGMENT}

The author would like to thank Clement Nadal for the useful discussions, FP7 Marie Curie Initial Training Network PROTOTOUCH, grant agreement No. 317100 for founding and IRCICA laboratory for hosting. 


\section{REFERENCES}

[1] M. Wiertlewski, J. Lozada and V. Hayward, "The Spatial Spectrum of Tangential Skin Displacement Can Encode Tactual Texture ," IEEE TRANSACTIONS ON ROBOTICS , vol. 27, no. 3, pp. 461-472, June 2011.

[2] M. Biet, F. Giraud and B. Lemaire-Semail, "Squeeze Film Effect for the Design of an Ultrasonic Tactile Plate," IEEE Transactions on Ultrasonics, Ferroelectrics, and Frequency Control, vol. 54, no. 12, pp. 2678-2688, Dec. 2007.

[3] L. Winfield, J. Glassmire, J. E. Colgate and M. Peshkin, "T-PaD: Tactile Pattern Display through Variable Friction Reduction ," EuroHaptics Conference, 2007 and Symposium on Haptic Interfaces for Virtual Environment and Teleoperator Systems. World Haptics 2007. Second Joint, pp. 421-426, 2007.

[4] W. Ben Messaoud, B. Lemaire-Semail, M.-A. Bueno, M. Amberg, and F. Giraud, "Closed-Loop control for Squeeze Film Effect in Tactile Stimulator," in Proceedings of the 2014 international Conference and exhibition on new actuators and drives, Actuator 2014, Bremen, 2014.

[5] E. Vezzoli, M. Amberg, F. Giraud and B. Lemaire-Semail, "Electrovibration modelling analysis," Eurohaptics 2014.

[6] K. A. Kaczmarek, K. Nammi, A. K. Agarwal, M. E. Tyler, S. J. Haase, and D. J. Beebe, "Polarity Effect in Electrovibration for Tactile Display ," IEEE Transaction on Biomedical Engineering, vol. 53, no. 10, pp. 2047-2054, October 2006.

[7] E. Mallinckrodt, A. L. Hughes, and W. Sleator Jr., "Perception by the Skin of Electrically Induced Vibrations," Science, pp. 277-278, Sep. 1953.

[8] O. Bau, I. Poupyrev, A. Israr, and C. Harrison, "TeslaTouch: electrovibration for touch surfaces ," in Proceedings of the 23nd annual ACM symposium on User interface software and technology, New York, 2010, pp. 283-292.

[9] M. Amberg, F. Giraud, B. Lemaire-Semail, P. Olivo, G. Casiez and N. Roussel, "STIMTAC, a Tactile Input Device with Programmable Friction," in Proceedings of the 24th annual ACM symposium adjunct on User interface software and technology, Santa Barbara, 2011.

[10] F. Giraud, M. Amberg, and B. Lemaire-Semail, "Mergin two tactile stimulation principles: Electrovibration and Squeeze film effect.," in World Haptics Conference (WHC), 2013, Daejeon, 2013, pp. 199-203.

[11] T. Watanabe and S. Fukui, "A method for controlling tactile sensation of surface roughness using ultrasonic vibration," in IEEE International Conference on Robotics and Automation 1995. Proceedings , vol. 1, 1995 , pp. 1134-1139.

[12] J. Meyer, M. Wiertlewski, M. A. Peshkin, and J. E. Colgate D., "Dynamics of Ultrasonic and Electrostatic Friction modulation for Rendering Texture on Haptic Surfaces," in proceedings of Haptics Symposium 2014, Houston, 2014, pp. 63-67.

[13] U. Abel, C.-D. Garthe, and a. K. H. Fruhstorfer, "Thickness of the Stratum Corneum of the Volar Fingertips ," Clinical Anatomy , no. 13, pp. 429-433, 2000.

[14] M. Wiertlewski and V. Hayward, "Mechanical Behavior of the Fingertip In The Range of Frequencies and Displacements Relevant to Touch ," Journal of Biomechanics, vol. 11, no. 45, pp. 1869-1874, 2012.

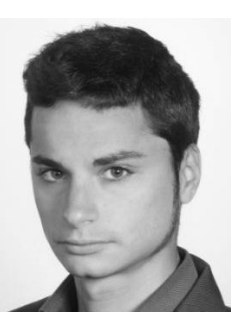

Eric Vezzoli got his MS degree in Physics Engineering from the Politecnico of Milan (Italy) in 2013. He has been research assistant at ESRF - European Synchrotron Radiation Facility to develop high-pressure cells for multi-extreme conditions studies. From September 2013 he is research assistant at L2EP-IRCICA Laboratory working on his $\mathrm{PhD}$ Thesis with the ITN Prototouch Project. His domain of research are tactile stimulation principle modelling, tactile display designing and tactile perception.

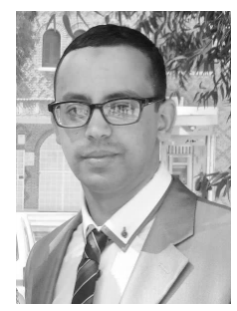

Wael Ben Messaoud was born in Sfax, Tunisia, in 1988. He received his M.Eng. degree in electrical engineering in 2011 and his MS degree in Automatic control and Industrial Computing in 2012 from the National School of Engineers of Sfax ENIS (Tunisia). He is currently PhD student in electrical engineering from November 2012. His thesis is in collaboration between L2EP Lab. University Lille 1 and LPMT Lab. University of Haute Alsace, France. His work is focused on haptic feedback device research.

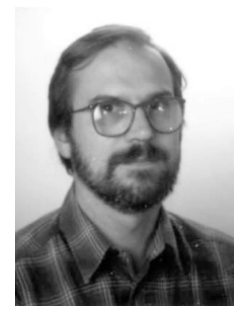

Michel Amberg has been teaching electronics at the University of Lille, France. He graduated from the Ecole Normale Supérieure de Cachan, France in 1981. He has tutored more than a hundred bachelor's students during their projects in the field of telecommunications, computer science, and electronics. He is now a research engineer at IRCICA, and works on the electronic design of tactile devices.

Frédéric Giraud received the B.s. degree in 1995 from Paris-XI University, graduated from the Ecole normale supérieure de cachan, France in 1996 in electrical engineering, and received the M.s. degree in 1997 from the Institut national Polytechnique de Toulouse and the Ph.d. degree in 2002 from the University lille 1. He is a mem- ber of the electrical engineering and power elec- tronics laboratory of lille, where he works as an associate Professor. His research deals with the modeling and control of piezoelectric actuators.

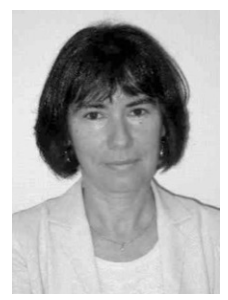

Betty Lemaire-Semail received the Ph.D. degree in 1990 from the University of Paris XI, Orsay. From 1990 to 1998 , she was an assistant professor at the Ecole Centrale of Lille and she is now a professor at the University Lille 1 . She is a member of the electrical engineering and power electronics laboratory of Lille and head of the research axis on the control of electrical systems. She has studied electromagnetic motors, and her main field of interest now deals with the modeling and control of piezoelectric actuators for positioning and force feedback applications.

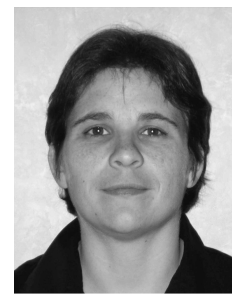

Marie-Ange Bueno received her $\mathrm{PhD}$ degree from the University of Haute Alsace France in 1995. Since 1997, she has been an assistant professor in fiber science and mechanics, and since 2005 a full professor at the Ecole Nationale Supérieure d'Ingénieurs Sud Alsace. She has worked since 1992 on tribology of fibrous materials with a special interest to tactile feeling. She is member of the French Mechanical Society (AFM) and the Fiber Society. 